CIUDAD Y TERRITORIO

ESTUDIOS TERRITORIALES

ISSN(P): 1133-4762; ISSN(E): 2659-3254

Vol. LII, № 204, verano 2020

Págs. $211-228$

https://doi.org/10.37230/CyTET.2020.204.02

CC BY-NC 4.0

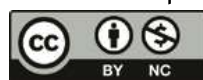

\title{
La cuantificación metropolitana en España. Hacia una definición homogénea de los espacios de gobernanza supramunicipal
}

\author{
Juan Ramón Selva-Royo
}

Departamento de Urbanística y Ordenación del Territorio. Universidad Politécnica de Madrid (España)

RESUMEN: Recientemente, han visto la luz en nuestro país diversos estudios sobre el hecho urbano metropolitano. A la vista del renovado interés por el análisis supramunicipal, parece conveniente profundizar en las propuestas de caracterización homogénea que recorren la literatura científica internacional y en su posible aplicación al caso español. Se estudian a continuación las metodologías cuantitativas basadas en la observación remota y en la información censal, así como las técnicas disponibles para la medición de la accesibilidad metropolitana. El proceso de validación de estos acercamientos -complementarios entre sí- queda delineado con el caso de estudio de las dos principales ciudades españolas, Madrid y Barcelona, a través de un análisis comparativo de su espacio periurbano. Por último, se abordan las consideraciones contemporáneas sobre la gobernanza territorial y el planeamiento metropolitano, surgidas del diálogo interdisciplinar entre urbanistas, geógrafos y economistas. Una serie de propuestas sobre la definición homogénea de los ámbitos supramunicipales, ligadas a bases de datos de acceso abierto y carácter global, completan esta mirada actualizada sobre las metodologías de delimitación de las áreas y regiones metropolitanas en España.

PALABRAS CLAVE: Áreas metropolitanas; Grandes áreas urbanas; Área urbana funcional; Gobernanza territorial; España.

\section{A quantitative approach to Spanish metro areas. Towards a homogeneous definition of supra-municipal planning scope}

ABSTRACT: Recently, several studies on urban metropolitan agglomerations have come to light in our country. In view of the renewed interest in supramunicipal analysis, it seems appropriate to deepen the homogeneous characterization proposals that run through the international scientific literature and its possible

Recibido: 04.02.2019; Revisado: 13.03.2019

Correo electrónico: juanramon.selva@upm.es NºRCID: https://orcid.org/0000-0001-6617-5741

El autor agradece los comentarios y sugerencias realizados por los evaluadores anónimos, que han contribuido a mejorar y enriquecer el manuscrito original. 
application to the Spanish case. The quantitative methodologies based on remote observation and census information are then studied, as well as the techniques available for the measurement of metropolitan accessibility. The validation process of these approaches - complementary to each other - is delineated with the case study of the two main Spanish cities, Madrid and Barcelona, through a comparative analysis of their peri-urban space. Finally, contemporary considerations on territorial governance and metropolitan planning, arising from the interdisciplinary dialogue between urbanists, geographers and economists, are addressed. A series of proposals on the homogeneous definition of supramunicipal areas, linked to open access global databases, complete this updated view on the delimitation methodologies of metropolitan areas and regions in Spain.

KEYWORDS: Metropolitan areas; Greater urban areas; Functional urban areas; Governance; Spain.

\section{Introducción: ¿una nueva caracterización urbana?}

n los últimos meses se han hecho públicos
diversos estudios con datos actualizados,
tanto nacionales como globales, sobre el
hecho urbano metropolitano. Es el caso del in-
forme Áreas Urbanas en España 2018, del Mi-
nisterio de Fomento, que utiliza el Padrón Muni-
cipal de Habitantes de 2017, el mapa de ocu-
pación del suelo correspondiente al proyecto eu-
ropeo Corine Land Cover, versión de 2018 (ya
disponible en el Centro de Descargas del CNIG),
o el detallado Atlas of the Human Planet 2018,
del Joint Research Center (JRC) de la Comisión
Europea.

Sobre todo, a la vista de este último documento, parece ya cercano el momento en el que la observación remota a partir de imágenes satelitales -combinada con la información censal desagregada y la interacción con los datos de movilidad en tiempo real- llegue a determinar de forma homogénea y precisa el alcance de nuestras metrópolis. Más aún, en un contexto de convergencia europea (al menos estadística o cuantitativa) cabe preguntarse si no se estarán sentando las bases para la incorporación de un nuevo enfoque que complemente aquellas caracterizaciones vinculadas a los límites administrativos.

Es claro que en España existen unas competencias claras asignadas a las entidades locales, y

\footnotetext{
${ }^{1}$ Este concepto de grado de urbanización ha sido refinado para superar la inicial asignación de la clasificación, vinculada al ámbito de las unidades administrativas locales en el segundo nivel (LAU2). Como es sabido, las LAU2s son de muy difícil comparación entre las diversas realidades nacionales e incluso regionales. Los centros urbanos caracterizados en el GHSL-SMOD de 2018 se definen como "la agrupación
}

que ya se dan diversas fórmulas de gobernanza para que el planeamiento se ajuste a la realidad urbana (Farinós Dasí \&, Peiró \& Gomis Fons, 2018: 175). Pero no deja de ser también patente que el alcance territorial de la mayor parte de los municipios hace tiempo que se vio superado por la expansión urbana, y que, en la práctica, no siempre es fácil coordinar la interfaz localregional en el ámbito político (HILDENBRAND, 2017: 37-42).

El presente artículo pretende realizar, junto con el repaso del estado del arte de los últimos avances metodológicos globales, un somero estudio comparativo de la caracterización de las áreas metropolitanas españolas (ver FIG. 1). Este acercamiento interdisciplinar aspira, por tanto, a contribuir a una reflexión tranquila sobre el desfase espacial del planeamiento tradicional en España, con el fin de formular preguntas que, no por arriesgadas, dejan de ser hoy en día menos oportunas.

\section{Definición reciente de las áreas urbanas}

\subsection{Técnicas de observación remota}

La teledetección y los procesos de observación remota global -liderados por el Group on Earth Observation-son, sin duda, campos que han experimentado un gran desarrollo en los últimos años (SABO \& al., 2018). Especialmente reseñable por

según criterios espaciales (High Density Clústers, HDC) de las celdillas de $1 \mathrm{~km}^{2}$ con una densidad poblacional de, a menos, $1.500 \mathrm{hab} / \mathrm{km}^{2}$, o de las que tengan un mínimo de $50 \%$ de su superficie construida (built-up), siempre que tengan un total de población superior a los 50.000 habitantes". Se establece además un criterio de contigüidad sencillo (de 4 celdas con relleno de huecos) 

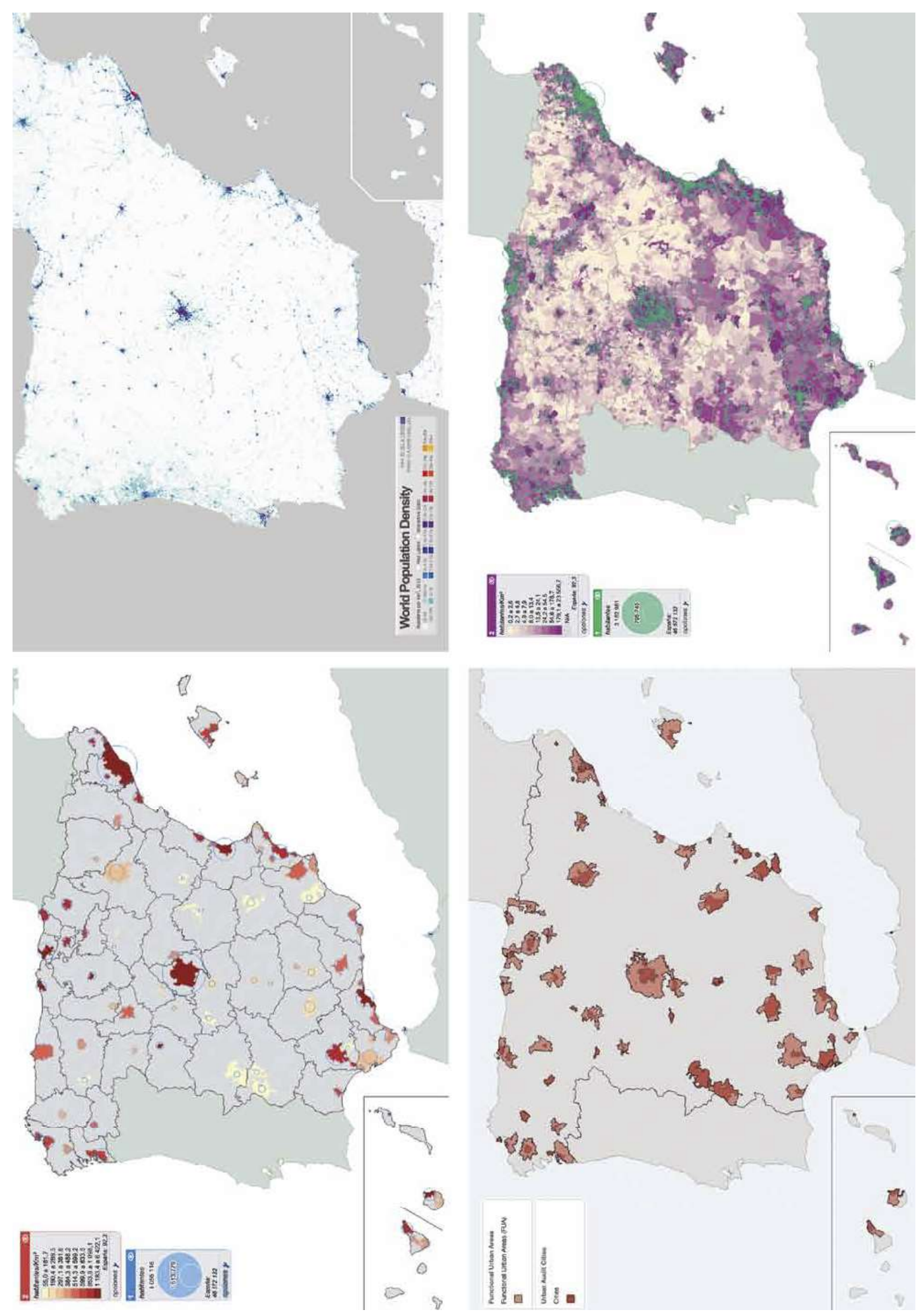

FIG. 1/ Caracterizaciones metropolitanas recientes: arriba, Grandes Áreas Urbanas, y mapa de densidades; abajo, Áreas Urbanas Funcionales según la OCDE; y visualización de densidades y población utilizando datos padronales de 2017 
su actualidad es el proyecto europeo Global Human Settlement (GHSL), responsable desde hace una década de la elaboración de información espacial de alta resolución bajo los principios del análisis cuantitativo directo, replicabilidad y acceso abierto (MELCHIORRI \& al., 2018).

Al igual que sus predecesores, la versión del atlas urbano de diciembre de 2018 de la Comisión Europea combina la información satelital -áreas construidas, espacios verdes e iluminación nocturna- con los datos censales de población procesados (CIESIN, 2017), para alumbrar un modelo de asentamientos (S-MOD, por sus siglas en inglés) basado en el concepto de grado de urbanización propuesto hace pocos años por DIJKSTRA \& PoELman (2014) ${ }^{1}$.

Pero ante todo, este atlas aporta un proceso de automatización en la clasificación supervisada mediante técnicas de inteligencia artificial Symbolic Machine Learning, SML, que da lugar a una nueva base de datos de centros urbanos (UCDB, de aquí en adelante) a escala planetaria con una gran resolución (ver FIG. 2). Es la primera vez que se agregan datos de los programas de observación remota Landsat y Sentinel-1, ampliándose además el umbral mínimo de población, habitualmente limitado a escala global a los 300.000 habitantes (UNITED NATIONS, 2018), hasta los 50.000. De esta forma, se enfoca directamente al cumplimiento de los indicadores ligados al objetivo $n^{\circ} 11$, Ciudades y comunidades sostenibles, de la Nueva Agenda Urbana fijada en Quito en 2016 (SENNETT \&, BURDETT \& SASSEN, 2018).

Se obtiene así una detallada información 4-D, con datos espaciales (localización y atributos), formales (extensión), agregados (población, densidad, espacios verdes, etc.) y temporales. Aunque cada capa trabaja con diferentes resoluciones, para la UCDB se asume una malla global de $1 \mathrm{~km}^{2}$ donde las celdas reciben la clase rural, suburbano, urbano o centro urbano según el S-MOD. Esto permite una consideración homogénea, no sólo desde el punto de vista poblacional o extensivo (SMITH, 2017: 117), sino para otras muchas variables socioeconómicas y ambientales (EUROPEAN Commission, 2018: 43-112).

\subsection{Criterios de accesibilidad}

Sin embargo, para que una delimitación metropolitana sea completa, no basta con una información estática, por muy detallada que sea. Como se ha recordado recientemente (METREX, 2014), es preciso considerar también las relaciones urbano-rurales de dependencia funcional de los grandes servicios, así como los datos de movilidad, fundamentalmente la ligada al ámbito laboral, denominada commuting (HORNER, 2004), y la movilidad residencial o migración (Romaní \& CASADO-Díaz \& LILLOBAÑULS, 2017: 708).

En este sentido, cabe destacar la investigación llevada a cabo por la Cátedra de Geografía Humana de la Universidad Pablo de Olavide, Sevilla (FERIA TORIBIO, 2018), desde donde se han realizado recientes propuestas de caracterización de las áreas metropolitanas de nuestro país (ver FIG. 3).

Atendiendo a la evolución de su delimitación histórica reciente, hay que reseñar la definición del sistema metropolitano español (FERIA TORIBIO \& Martínez Bernabeu, 2016), que ha merecido ser incluida como mapa temático del Atlas Nacional
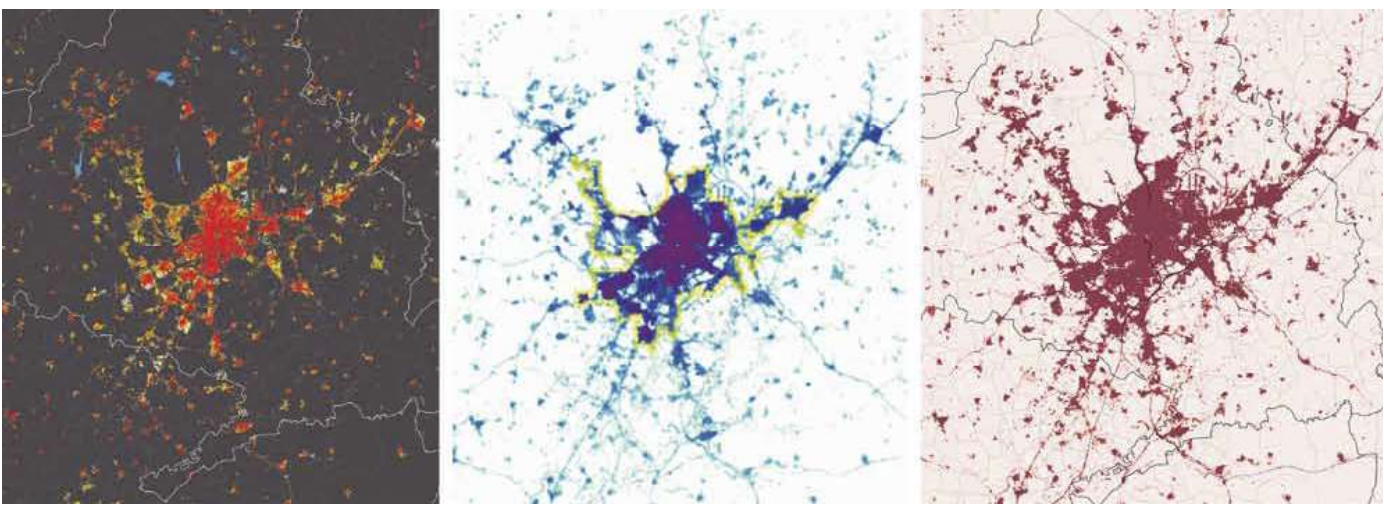

FIG. 2/ Imagen multi-temporal 1975-1990-2000-2015 de Madrid para la capa built-up, superficie construida; visualización de los High Density Clusters (HDC) del centro urbano de Madrid; y densidad de población con paso de celda de $250 \mathrm{~m}$

Fuente: Elaboración propia sobre datos del GHSL-SMOD de 2016; SMITH, 2017; y CIESIN (2017), respectivamente. 


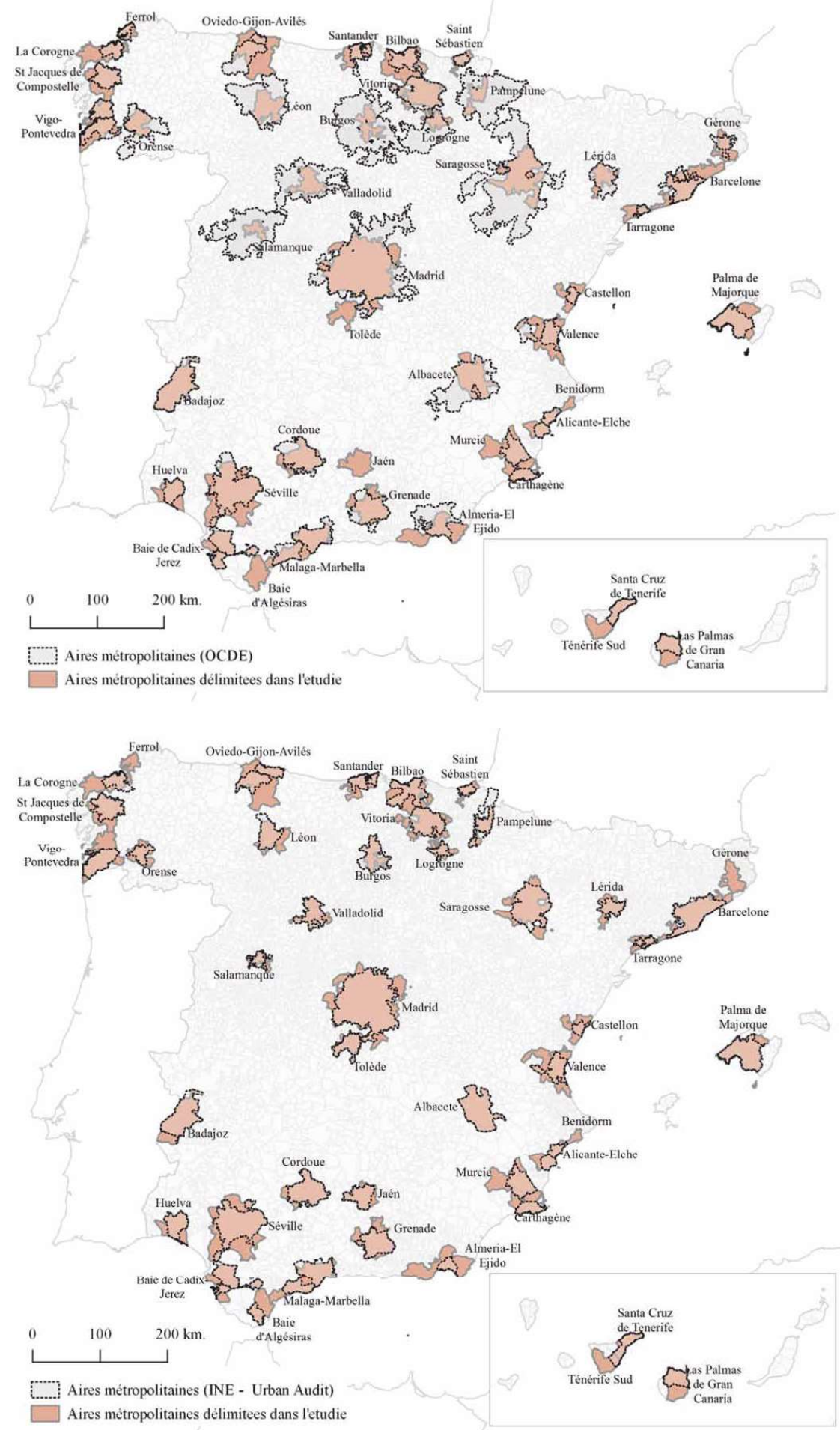

FIG. 3/ Mapas comparativos del sistema metropolitano español con datos de 2011 (en color), y las delimitaciones derivadas de la aplicación de criterios de la OCDE de 2012 (arriba) y del INE - Urban Audit 2016 (abajo).

Fuente: Feria Toribio \& De Oliveira Neves \& Hurtado Rodriguez, 2018: 18-19. 
de España, ANE (INSTITUto Geográfico NACIONAL, 2018: 216), así como sus respectivas comparativas con la delimitación derivada de los criterios de la OCDE de 2012 y con los datos del INE de 2016 (Feria Toribio \& de Oliveira Neves \& Hurtado Rodríguez, 2018) 2 .

Si bien las definiciones metropolitanas se realizan fundamentalmente como referencia espacial para la estadística pública y no a efectos de gobernanza, esta aproximación presenta, a nuestro entender, y a pesar de su metodología iterativa basada en una valiosa información estadística, dos escollos para su ajuste fino. Por un lado, se depende en muy alto grado de los datos censales desagregados -estructura de la población, movilidad laboral, etc.- algunos sólo disponibles cada diez años (el censo utilizado en el mapa del ANE 2018 es de 2011); y por otro, se parte del presupuesto irrenunciable de considerar la delimitación final como una mera agregación de términos municipales contiguos

Por contraposición, el recurso a la celda de $1 \mathrm{~km}^{2}$ podría ser rechazado argumentando que los datos censales y de movilidad de que se dispone están habitualmente recopilados por término municipal, además de que una delimitación final no estrictamente coincidente con las entidades locales carecería de sentido a la hora de trasponerla al ámbito urbanístico (cuyas competencias recaen en los ayuntamientos, y mediante las cuales todo el suelo incluido en cada municipio recibe una clasificación).

La primera objeción puede rebatirse si se consideran las fuentes empleadas por el JRC europeo: los censos de la población mundial, GPW v4.10, así como su proyección e interpolación estadística coordinada por la Universidad de Columbia (CIESIN, 2017), son actualizados cada año sobre las estimaciones de las Naciones Unidas, basadas a su vez en los últimos censos nacionales e información padronal disponibles.

Además, en la versión de 2018 se ha mejorado la integración de las capas de superficie construida (GHS-BU, con resolución de $20 \mathrm{~m}$ ) y de población (GHS-POP, con resolución de $250 \mathrm{~m}$ ), razón por la que las áreas urbanas se han podido afinar hasta niveles sin precedentes (EUROPEAN Commission, 2018).

En cuanto a la accesibilidad, cabe decir que desde diciembre de 2018 está disponible en acceso

\footnotetext{
2 El Atlas Nacional de España ha sido también recientemente incluido en la web del Centro Nacional de Información Geográfica (CNIG), dentro del apartado de Información Geográfica Temática/Mapas temáticos del ANE, para su libre acceso público en la siguiente dirección:
}

abierto un mapa global con respecto a todo centro urbano definido por el GHSL, fruto de un reciente estudio liderado por la Universidad de Oxford (WeIss \& al., 2018). Con un tamaño de celda de $1 \mathrm{~km}^{2}$, se ha podido establecer el tiempo estimado de viaje desde todo punto a cada uno de los 13.840 High Density Clusters (HDC) globales, mediante la combinación de la red de carreteras de OpenStreetMap y un algoritmo de proximidad derivado de la base de datos de Google Maps (ver FIG. 4).

Aunque esta información no cubre toda la complejidad del fenómeno urbano de la movilidad -ni tiene en consideración la red ferroviaria ni los problemas de congestión, entre otros aspectos-, supone un paso extraordinario para acceder a información homogénea y altamente actualizada (y en un futuro previsiblemente dinámica). Es más, en el caso particular de España, los HDC considerados en ese mapa se han construido teniendo en cuenta la estimación de población calculada en 2015 por el CIESIN, lo que supone una considerable mejora con respecto a la metodología para obtención de información metropolitana disponible hoy en día en nuestro país (MINISTERIO DE Fomento, 2018: 20).

Finalmente, con respecto a la segunda objeción antes apuntada (la dependencia última de los términos municipales para la delimitación de las áreas metropolitanas) ${ }^{3}$, cabría preguntarse si no son éstos, es decir, los municipios, los que necesitarían una urgente actualización espacial... Como es obvio, una propuesta de este calado ha de tener en cuenta otros muchos factores socioculturales y políticos, por lo que remito a la discusión final del artículo para su consideración sosegada.

En cualquier caso, la exigencia de contigüidad para el alcance metropolitano, aun obviada en algunas metodologías recientes (UCHIDA \& NELSON, 2011), parece derivarse tanto de los presupuestos metodológicos del GHS-SMOD como del trabajo de WEISS \& al. (2018). Sin embargo, en un escenario de reformulación dinámica de las entidades locales, no hay que dejar de señalar que ese no debería ser un problema, pues muchos de los municipios españoles ya cuentan hoy con enclaves, es decir, con territorios no contiguos rodeados de otros términos, provincias e incluso países (como son los casos, entre otros, de Valencia, Treviño o Llívia, respectivamente)

htp.//centrodedescargas cnig.es/CentroDescarcas/index isp\# ${ }^{3}$ Este criterio es seguido también por el Ministerio de Fomento a la hora de definir las Grandes Áreas Urbanas (GAUs), a saber, aquellas que suman más de 50.000 habitantes, para el documento publicado en diciembre de 2018. 


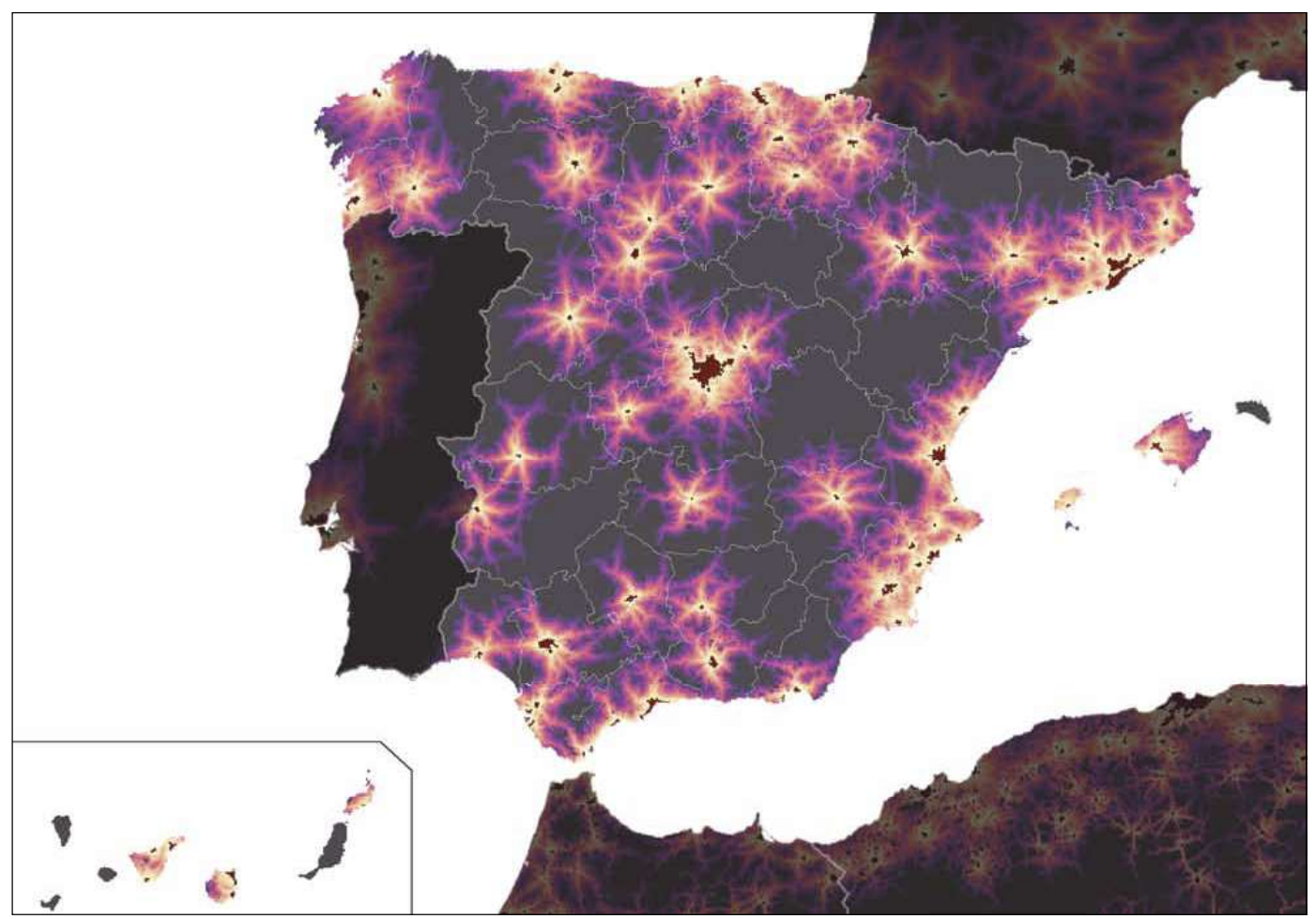

FIG. 4/ Mapa de España con indicación de los HDC (áreas urbanas) según el GHS-SMOD de 2016, sobre gráfico de isócronas de menos de una hora a cada centro urbano.

Fuente: Elaboración propia a partir de datos de Pesaresi \& Freire, 2016; Pesaresi \& al., 2016, y Weiss \& al., 2018.

\section{Caso de estudio: dos metrópolis españolas}

Según la Urban Center Database, UCDB R2019A, de la Comisión Europea, en España se contabilizan hasta 73 centros urbanos (UC) que cumplen con las condiciones definidas por DIJKSTRA \& POELMAN (2014), dándose la particularidad de que cuatro de ellos son transfronterizos: Algeciras-Gibraltar, Ceuta-Fnideq, Melilla-AitMansor e Irún-Biriatou.

Por otra parte, el Atlas de Áreas Urbanas $2018 \mathrm{de}$ Ministerio de Fomento, que utiliza datos padronales de 2017, señala, exclusivamente para nuestro país, 86 Grandes Áreas Urbanas (GAUs) -es decir, de más de 50.000 habitantes-, ya sean uni o plurimunicipales. Como compilación complementaria, el sistema español de Áreas Metropolitanas según FERIA \& MARTINEZ (2016) se compone de 45 unidades, siempre con información del censo de 2011. Más allá del número exacto, y habida cuenta

${ }^{4}$ Estas dos ciudades lideran todas las clasificaciones apuntadas, pero difiriendo en metodología y delimitación final. Para acceder a su información desagregada por municipio de que ninguna de las tres relaciones es vinculante para el planeamiento urbanístico, interesa ahora centrar la mirada en el caso de estudio comparativo de las dos mayores e indiscutidas áreas metropolitanas españolas, Madrid y Barcelona ${ }^{4}$.

En primer lugar, en cada propuesta metodológica el número de municipios implicados varía notablemente (ver FIGs. 5 y 6). Así, la GAU de Madrid engloba 52 términos, por los 165 de la de Barcelona, muy distantes de los 172 y 130 propuestos respectivamente en el ANE. Como se ve, la demarcación de la capital aumenta sobremanera su número de municipios, mientras que el área catalana, utilizando la misma metodología, los pierde.

No se contabilizan los términos municipales englobados en la UCDB por trascender esta base de datos sus delimitaciones, pero puede apreciarse a simple vista que, en todo caso, las áreas urbanas son más reducidas. Es más, dentro de

pueden consultarse, respectivamente el Atlas of the Human Planet 2018, el Atlas de Áreas Urbanas 2018 y el Atlas Nacional de España 2018. 


\section{Estudios}

las áreas fijadas por FERIA \& MARTíneZ (2016), se pueden contar, para Madrid, dos GAUs -Madrid y Guadalajara-y cuatro HDC -Madrid, Collado-ViIlalba, Alcalá de Henares y Guadalajara- (según el cómputo afinado de 2018). Para Barcelona, y siempre sin tener en cuenta el planeamiento aprobado, el área metropolitana y su GAU correspondiente no coinciden espacialmente, si bien ambas incluyen tres HDC (aunque éstas difieren según la versión del atlas: en 2016 se trata de los centros urbanos de Barcelona, Vilanova i la Geltrú y Blanes, mientras que en 2018 éste último es sustituido por el de Mataró).

Junto a la metodología reseñada en cada publicación, basada, como es habitual, en datos poblacionales y de movilidad (GómEZ GIMÉNEZ, 2017: 5), existe otro factor que parece distorsionar las delimitaciones basadas en la unidad territorial municipal. En efecto, las GAUs establecidas por el Ministerio de Fomento se detienen siempre ante los límites provinciales, razón por la cual Madrid y Guadalajara, o Barcelona y Blanes-Lloret de Mar, no se fusionan en una sola Gran Área Urbana. A la vista de este doble caso de estudio, parece clara la complejidad a la que se enfrenta la delimitación metropolitana en España. Ateniéndose, en cambio, a cuestiones estrictamente cuantitativas, se observa que sí es posible establecer un ámbito espacial homogéneo, abierto a actualizaciones (incluso éste podría ser ligado, con posterioridad, a la competencia municipal correspondiente).

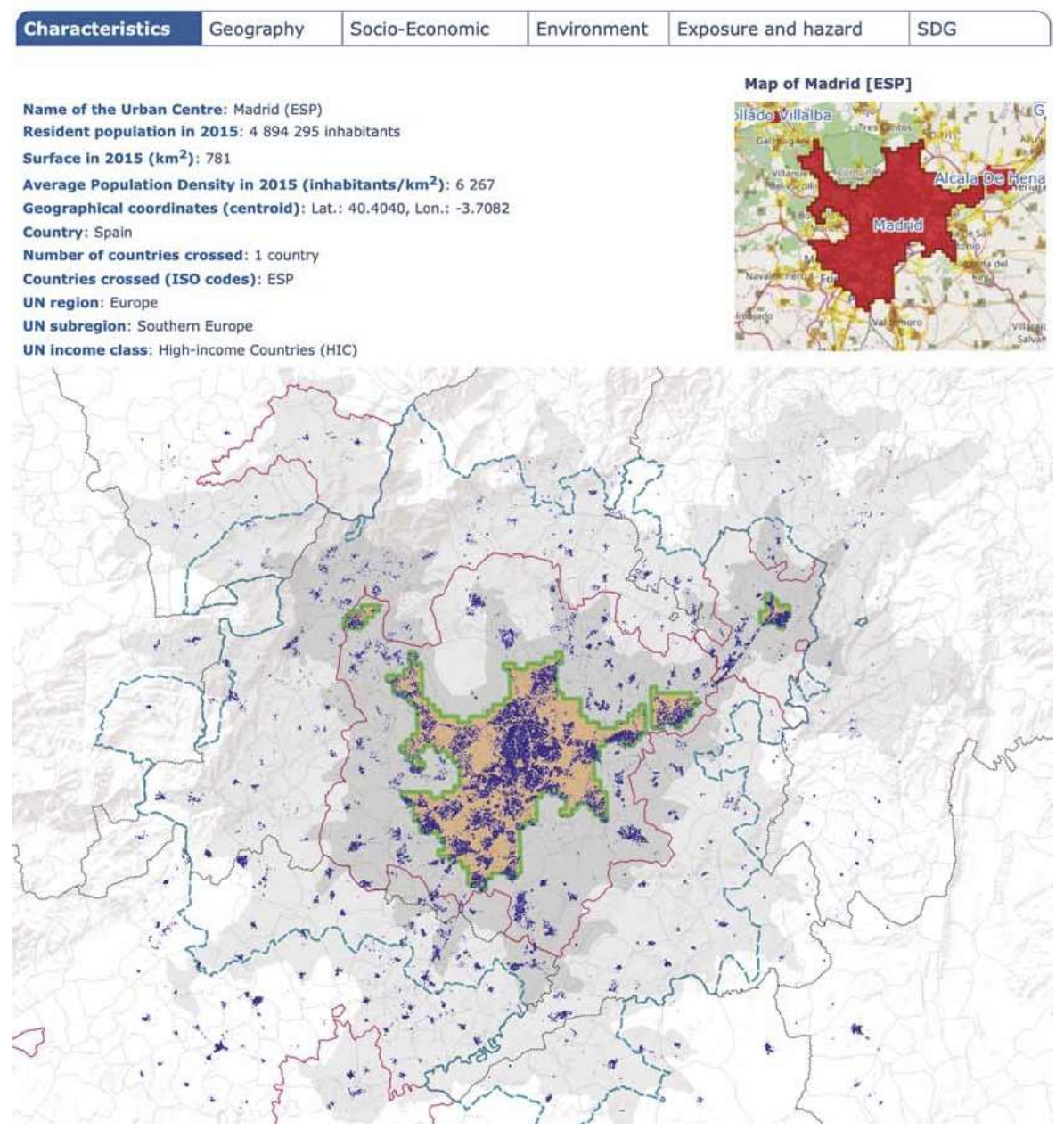

FIG. 5/ Área metropolitana de Madrid y ámbito actualizado del GHS-SMOD 2016 (isócronas de 10' y 30' en sombreado gris). En rojo, ámbitos de las GAUs; azul, Áreas Metropolitanas del ANE; y verde, centros urbanos (HDC) de 2018.

Fuente: Elaboración propia sobre datos de EUROPEAN COMMISSION, 2018; PESARESI \& FREIRE, 2016 Ministerio de Fomento, 2018; Weiss \& al., 2018; y Feria \& Martinez, 2016. 
La integración de la movilidad se puede fijar aumentando el alcance de los HDC según un tiempo prefijado -en los estudios del área urban economics suele utilizarse el de una hora (DURANTON, 2015)-, y combinarse a su vez con umbrales basados en datos estadísticos. Pero, teniendo en cuenta que el JRC incluye también datos socioeconómicos de cada centro urbano (nivel de ingresos, variación de población, etc.), el alcance bien podría remitirse a estudios recientes (MORENO-MONROY \& VENERI \& SCHIAVINA, 2019) que replican globalmente los criterios de commuting de la OCDE para la UCDB de 2019.

De esta forma, accederíamos a un atlas actualizado de Áreas Funcionales Urbanas (Functional Urban Areas, FUAs, por sus siglas en inglés), basado en la definición que la Comisión Europea otorga a los centros urbanos de, al menos, 50.000 habitantes rodeados de

"a less densely populated commuting zone whose labour market is highly integrated with the city" (EUROPEAN UNION, 2016).

¿Sería deseable que la delimitación de áreas metropolitanas en España se complementara con uno de estos modelos europeos de hinterland? Desde luego, sin abandonar la actual metodología, se facilitaría así la consideración homogénea del ámbito funcional de la ciudad real. Cuestión distinta es, no obstante, la postura que adopte el planeamiento metropolitano en cada país de acuerdo con su propia soberanía y su tradición administrativa.

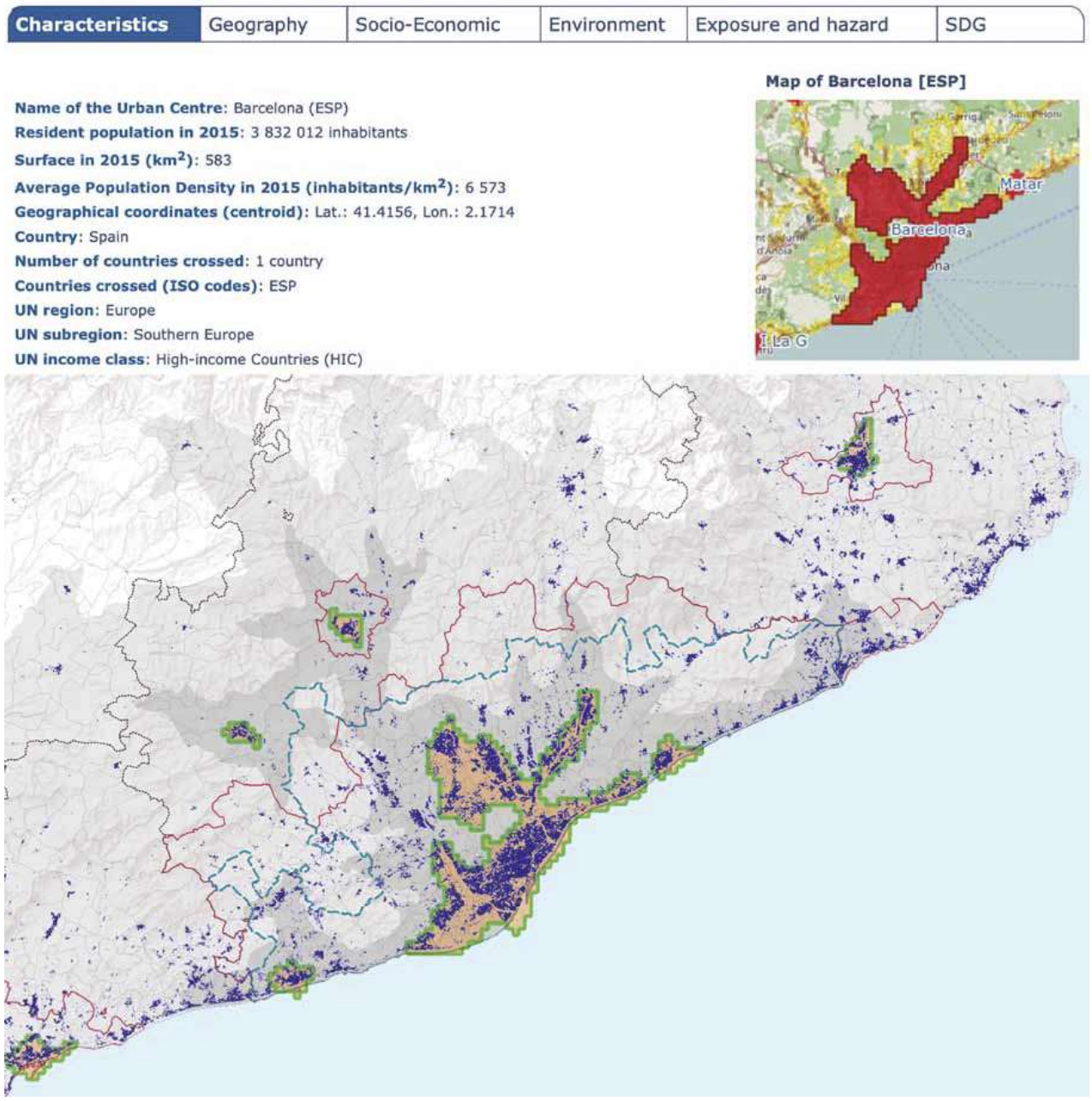

FIG. 6/ Área metropolitana de Barcelona y ámbito actualizado del GHS-SMOD 2016 (isócronas de 10' y 30' en sombreado gris). En rojo, ámbitos de las GAUs; azul, Áreas Metropolitanas del ANE; y verde, centros urbanos (HDC) de 2018.

Fuente: Elaboración propia sobre datos de EUROPEAN COMMISSION, 2018; PeSARESI \& FREIRE, 2016; Ministerio de Fomento, 2018; WeIss \& al., 2018; y Feria \& MARTinez, 2016. 


\section{El futuro del planeamiento metropolitano}

\subsection{Situación actual en España}

Los sistemas de planificación supramunicipal, como todo el planeamiento territorial en nuestro país, caen dentro del marco competencial de las Comunidades Autónomas. Incluso a nivel estatal, el artículo 25 de la Ley de Bases de Régimen Local remite la concreción de las competencias de planeamiento y gestión urbanística, que corresponden a los municipios, a la legislación sectorial pertinente: son, pues, las diferentes leyes de ordenación territorial o de urbanismo de cada autonomía las que concretan las competencias en materia de planeamiento.

A la vez, es conocida la desatención política sufrida por el planeamiento metropolitano en España (HILDENBRAND, 2017), así como las reiteradas llamadas a la innovación de la cultura del territorio en el actual contexto de gobernanza cambiante y sujeta a los avances del conocimiento (FARINós DAsí \& al., 2017).
Precisamente el Grupo de Investigación de Gobierno y Desarrollo Local Sostenible, de la Universidad de Valencia, ha efectuado recientemente una valiosa puesta al día de la planificación y ordenación territorial en espacios metropolitanos españoles (FARINós Dasí \& PEIRÓ \& Gomis Fons, 2018). Los diversos instrumentos subregionales han sido recogidos y relacionados con sus respectivos espacios metropolitanos (ver FIG. 7).

El trabajo señala que apenas 8 de ellos tienen un carácter integral o comprehensivo, $\mathrm{y}$, entre estos, son minoría los relativos a las metrópolis reseñadas con aprobación definitiva: el Plan Estratégico de Zaragoza y su Entorno, o el Plan Territorial Metropolitano de Barcelona -vinculante, por cierto, para todos los municipios incluidos en la respectiva $\mathrm{GAU}-$, son dos de sus principales exponentes.

Teniendo en cuenta la trascendencia de la planificación urbanística para la sostenibilidad del territorio -recientemente subrayada por la Agenda Urbana Española ${ }^{5}$-, así como la dificultad de los organismos autonómicos para trascender el ámbito local de competencias en materia de

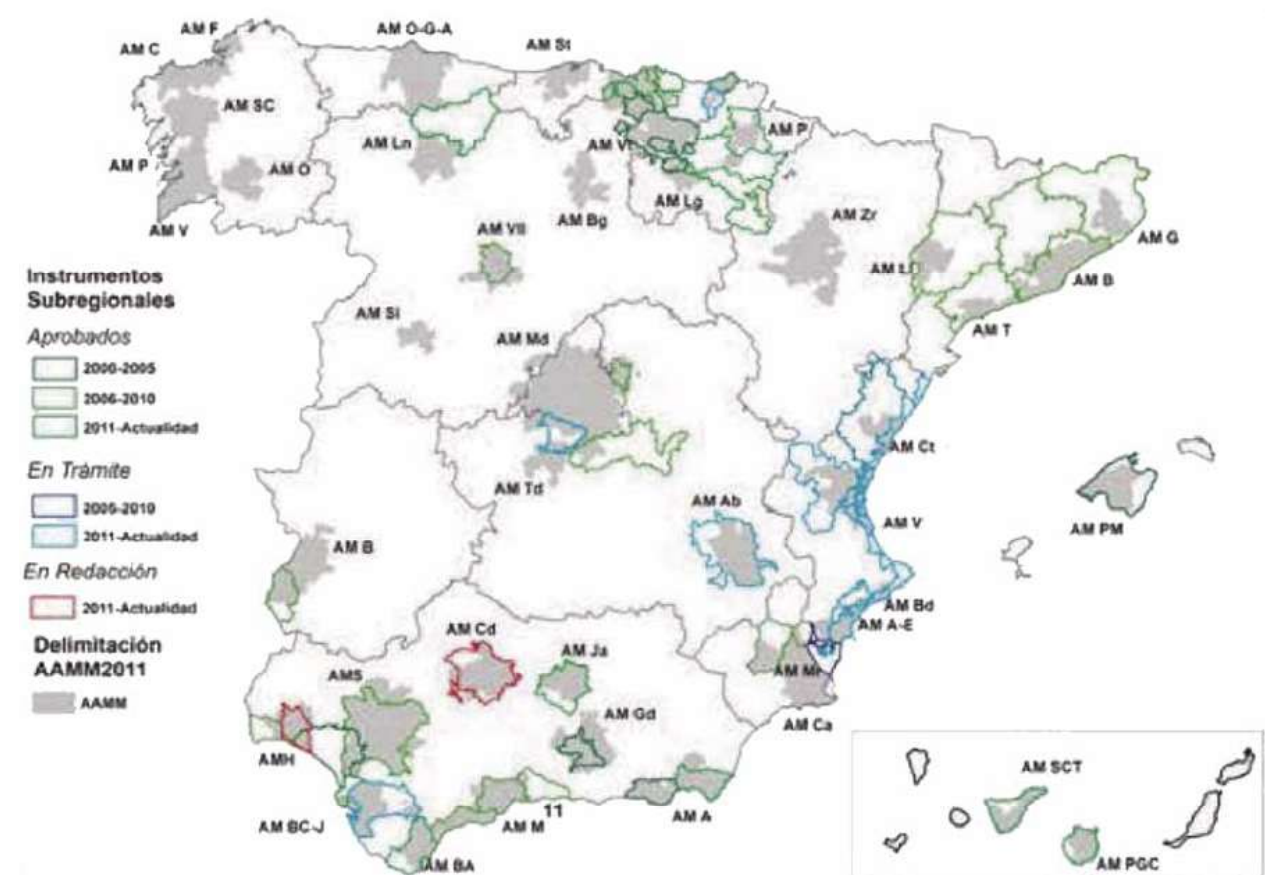

FIG. 7A/ Mapa de instrumentos de planificación subregional y áreas metropolitanas (información censal de 2011)

${ }^{5}$ La Agenda Urbana Española, presentada en febrero de 2019, es un documento de carácter estratégico sobre el futuro del modelo urbano nacional. El planteamiento territorial se erige ahí como uno de los principales responsables de varias de las líneas de actuación del Objetico Estratégico 2 (equilibrio urbano y funcional, ocupación eficiente del suelo, etc.) 


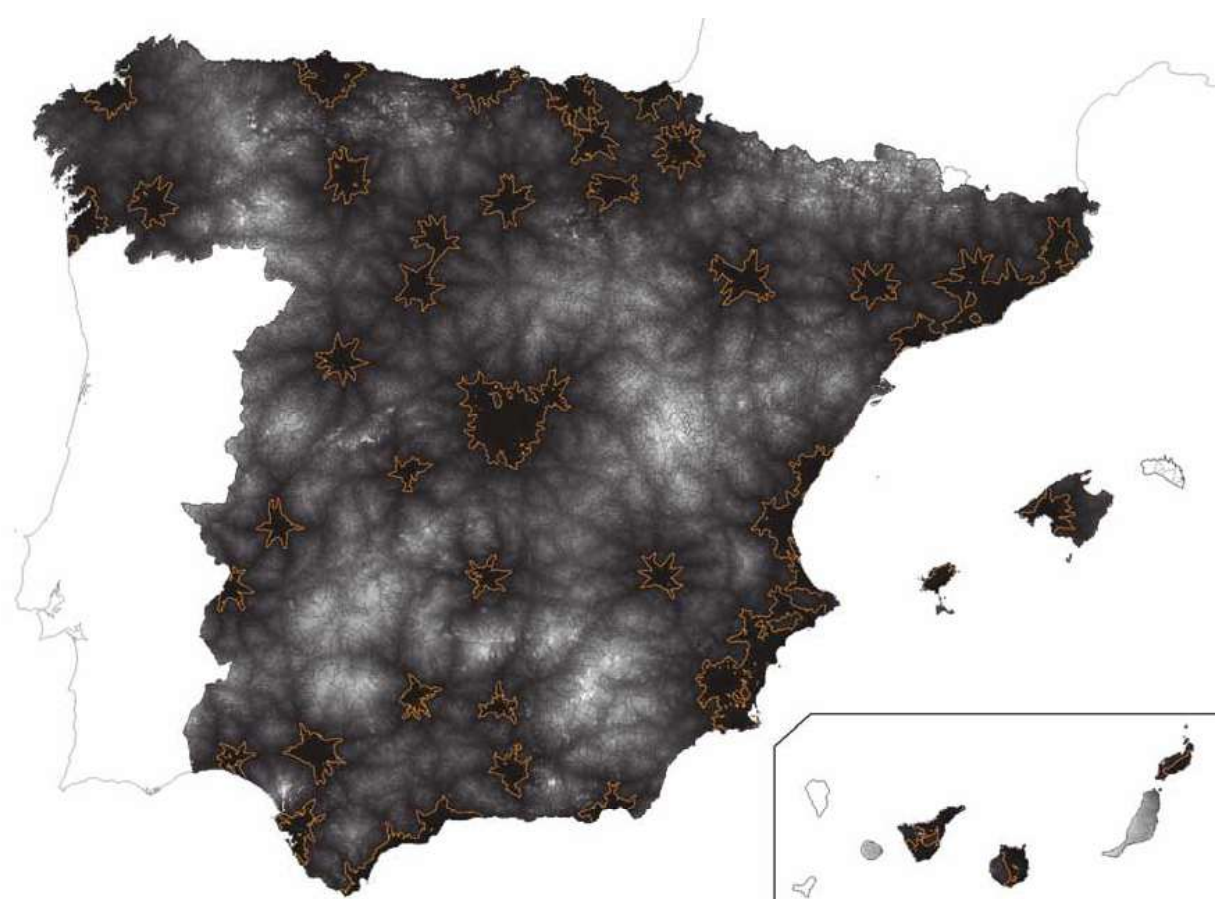

FIG. 7B/ Isócronas de 15 minutos a los HDC españoles definidos en el GHSL-SMOD de 2016, según cálculo de WEISS \& al. (2018)

Fuente: FARINós \& PEIRÓ \& Gomis, 2018: 179; y elaboración propia

clasificación de suelo o de vivienda de protección pública (fundamentales, por otra parte, para el equilibrio financiero de los municipios), sería muy deseable que los responsables regionales apostaran por el desarrollo de instrumentos de ordenación subregionales de carácter vinculante. Si además esos planes se centraren en los ámbitos metropolitanos, asumiendo un enfoque integral de las políticas económicas, ambientales y sociales, nuestras ciudades -incluso conservando parte de su especificidad legislativa y tradición urbanística- llegarían a ser mucho más competitivas en el actual contexto de convergencia europea (Metrex, 2014; Elinbaum \& Galland, 2016).

\subsection{Del píxel a la circunscripción administrativa}

Volviendo a la cuestión de la definición homogénea de los ámbitos del planeamiento metropolitano, el debate sobre cómo efectuar el salto de las caracterizaciones cuantitativas a los distritos administrativos está ya encima de la mesa (BosKER, PARK \& RoBERTS, 2018). De hecho, todas las caracterizaciones basadas en observación remota o clústers -se hayan obtenido por medio de aglomeración (DIJKSTRA \& POELMAN, 2014), flujos de movilidad (DURANTON, 2015) o iluminación nocturna (NOAA) - presentan la misma problemática de cómo quedar adscritas a un espacio concreto de gobernanza.

En la práctica, se suele aceptar la sencilla regla de considerar metropolitanas dos o más circunscripciones enteras si al menos el $50 \%$ de la población de cada una está afectada por la extensión urbana resultado de cada metodología (urban center o HDC, en nuestro caso). Considerando cómo están ligadas en la observación remota del GHSL las capas de superficie construída con las de población (disponibles hasta una resolución de rejilla de $250 \mathrm{~m}$ de lado), desde el punto de visto técnico esta cuestión no presenta mayor problema (FLORCZYK \& al., 2019b).

Sin embargo, y aun cuando esta opción justifique algunos estudios, el carácter dinámico y cada vez más concentrado de las áreas metropolitanas españolas (FERIA ToRIBIO, 2018: 653) hace que el recurso al municipio como unidad mínima indivisible deba ser complementado. Máxime cuando la dimensión de los términos municipales, imposible de ser reducida a patrones cuantitativos (precisamente por responder a otras realidades, 
históricas o culturales), suele distorsionar gravemente el ámbito final metropolitano, obligando, por ejemplo, a incluir grandes cantidades de superficie ante un leve cambio migratorio o poblacional en las condiciones iniciales.

Por el contrario, un eventual acceso a la información desagregada de movilidad urbana, combinado con tecnologías basadas en el sensing (Meyers \& Shaheen, 2017; Rode \& dA CRUz, 2018), permitiría afinar y corregir la dimensión supramunicipal con gran precisión y, en ocasiones, hasta en tiempo real. Además, tal y como se ha visto en el caso de estudio, esta aproximación se ajusta mucho mejor a la metrópolis polinuclear, en la que las distancias a los diferentes centros urbanos se solapan con tiempo inferior a 30 minutos (nótese que en la FIG. 5 el sombreado gris claro, correspondiente a la isócrona de media hora desde los HDC según medición sobre la base de datos de OpenStreetMap, sobrepasa con creces cualquier delimitación metropolitana previa).

\section{5. Áreas y regiones metropolitanas en España}

El fenómeno aludido de concentración urbana obliga a complementar la visión tradicional de las áreas metropolitanas. Más que hablar de una dependencia centrípeta (urban core), la gran cantidad de núcleos urbanos que quedan integrados al combinar la UCDB de la JRC (FLORCZYK \& al., 2019a) con las distancias obtenidas por WEISS \& al. (2018) para tiempos relativamente cortos, hace que en ocasiones resulte más propio referirse a redes o regiones metropolitanas.

Teniendo en cuenta la población disponible en el CIESIN (2017), y considerando homogéneo el reparto de la accesibilidad, la delimitación territorial de estos espacios quedaría supeditada al establecimiento de un rango convenido de distancias. En el presente estudio, se ha determinado el umbral de 30 minutos para las regiones metropolitanas, si bien podrían fijarse otros tiempos. En cambio, sí que parece adecuado que esta distancia se acorte para las áreas metropolitanas (se identifica una isócrona de 10 minutos) si con ello se pretende acotar un ámbito funcional más acorde a los instrumentos de planeamiento subregional de nuestro entorno (ver FIG. 8).

Otro factor determinante en esta caracterización es la no consideración de centro urbano, a partir del cual establecer un área de influencia, de aquellas poblaciones inferiores a los 50.000 habitantes (o, en sentido estricto, de aquellas que, con los datos actuales, no cumplen con los requisitos fijados por DIJKSTRA \& POELMAN (2014) para ser urban center). Es el caso, entre otros, de Santiago de Compostela o Toledo, ambas capitales autonómicas, lo que no deja de distorsionar la realidad funcional metropolitana. De cualquier forma, las densidades que arrojan los espacios así definidos resultan más homogéneos y ajustados a la realidad, al no considerar la parte deshabitada de los municipios (ver FIG. 9).

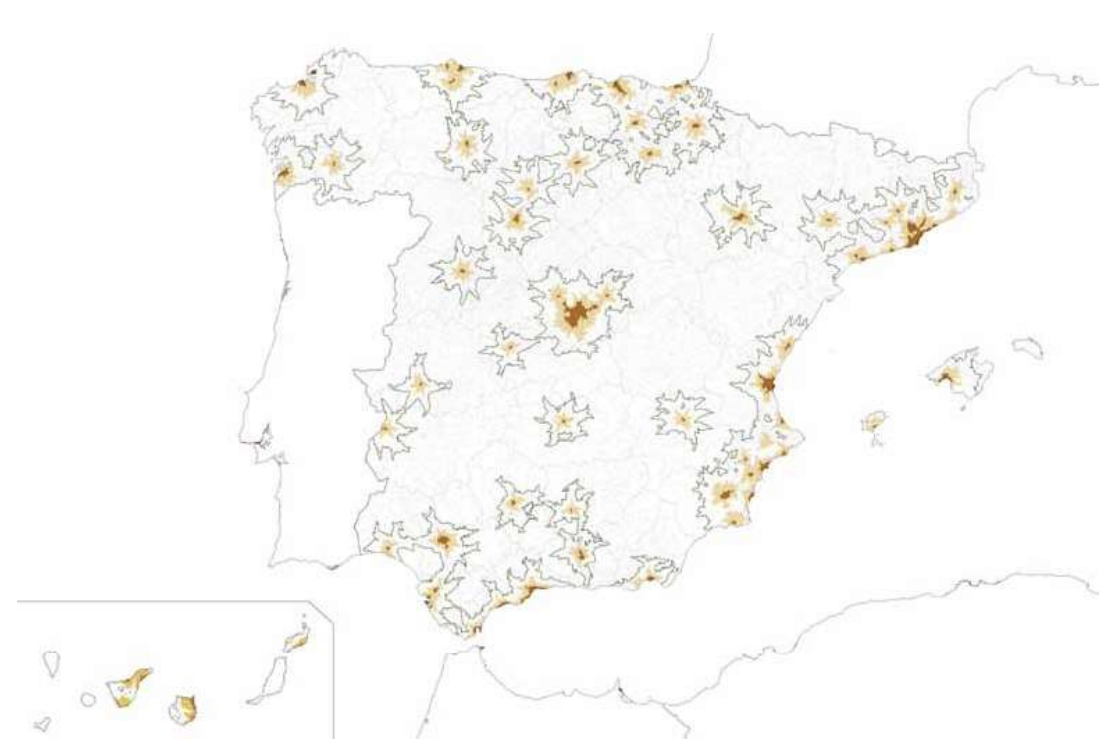

FIG. 8/ Mapa municipal de España base para las propuestas de delimitación de los espacios metropolitanos, con superposición de isócronas de 10 (relleno amarillo) y 30 minutos (en verde) desde cada centro urbano. En color oscuro, los HDC según el UCDB de 2019.

Fuente: Elaboración propia a partir de FLORCZYK \& al., 2019a. 


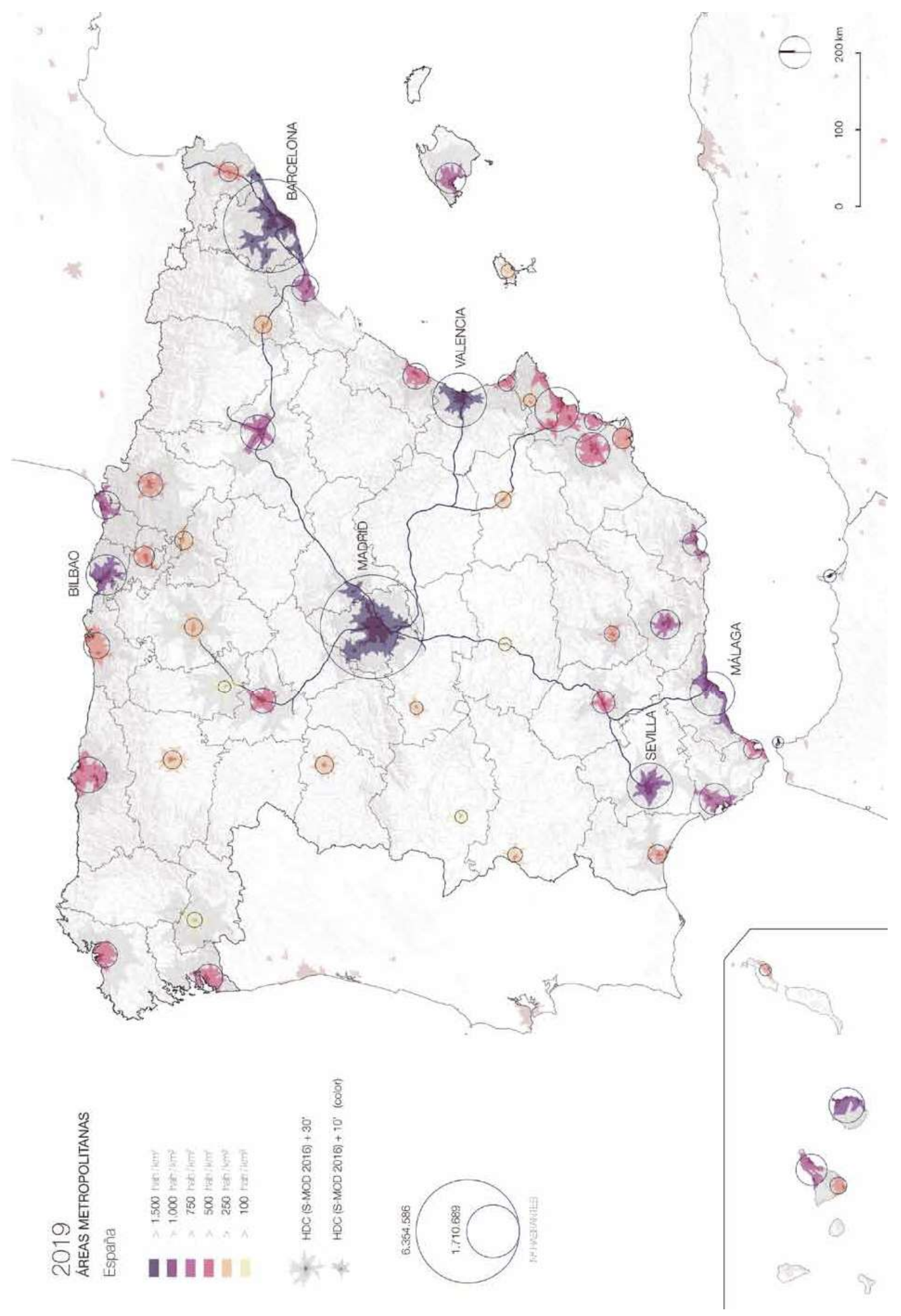

FIG. 9/ Propuesta de Áreas Metropolitanas para España, con los centros urbanos, HDC, del S-MOD de 2016, con isócronas de 10 minutos y escala de color según densidad de población.

Fuente: Elaboración propia a partir de CIESIN 2017 y FLORCZYK \& al., 2019a. 


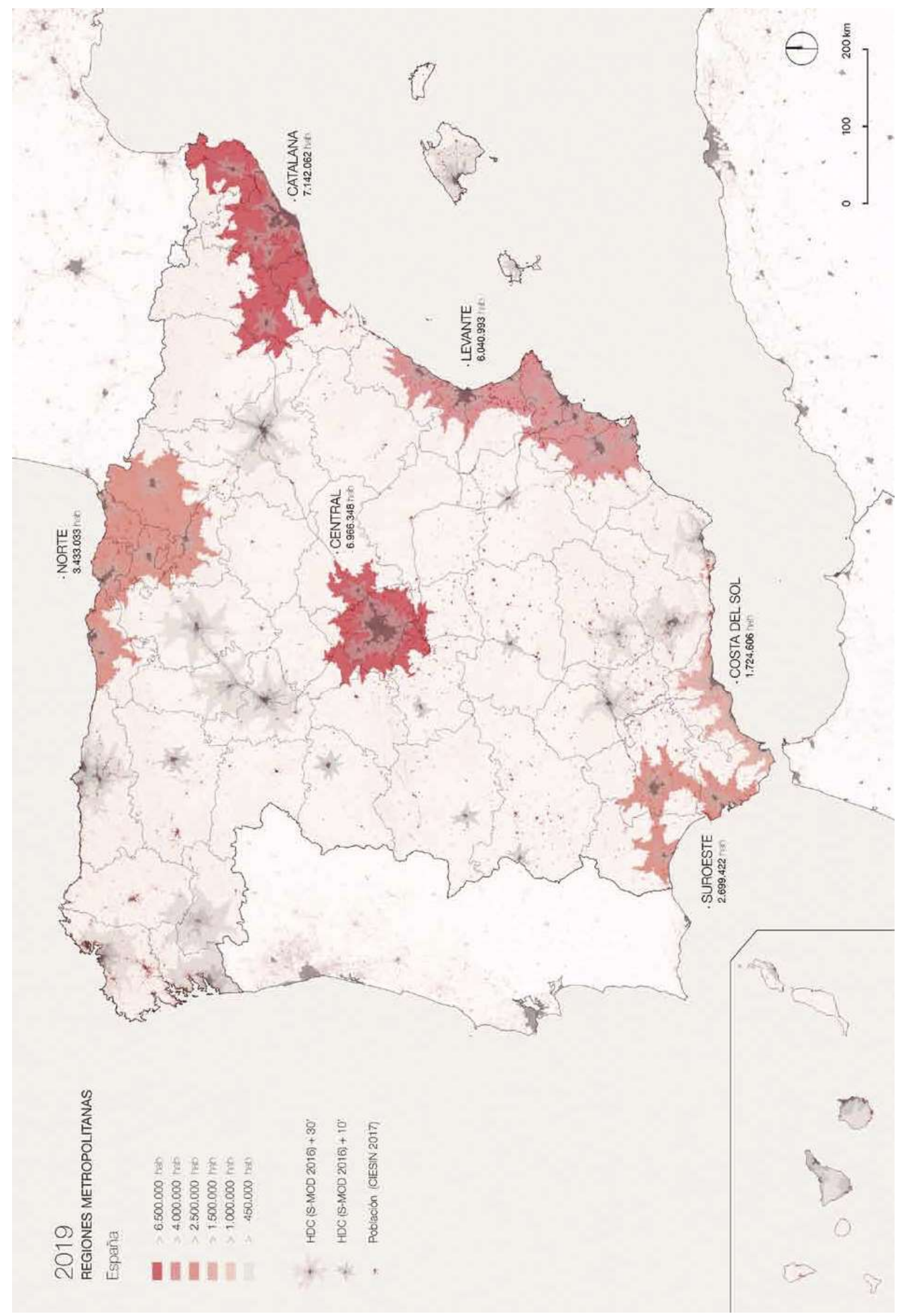

FIG. 10/ Mapa sugerido para las Grandes Regiones Metropolitanas, utilizando datos censales procesados y desagregados espacialmente, y HDC de 2018, con isócronas de 10 y 30 minutos y escala de color según número de habitantes.

Fuente: Elaboración propia a partir de CIESIN 2017 y FLORCZYK \& al., 2019a. 
Fijadas estas premisas, tendríamos para España un total de 51 áreas y 15 regiones metropolitanas (más de 500.000 habitantes). De estas últimas, siete tendrían la categoría de grandes regiones metropolitanas (GRM), entendiendo por tales las que superan en su conjunto el millón de personas. Como condición adicional para las regiones, se ha establecido que las isócronas de 30 minutos desde los centros urbanos o HDC estén suficientemente solapadas. En este sentido, la correspondiente a Vigo-Pontevedra-Orense sería el caso límite para la fusión de isócronas, mientras que las de Córdoba-Jaén-Granada o Asturias-León, aun sumando en su conjunto algo más de un millón de personas, no se incluirían como GRM por estar conectadas por corredores muy estrechos (ver FIG. 10).

Aun con todo, el resultado gráfico que arroja la metodología propuesta, desde el punto de vista estrictamente cuantitativo, se ajusta visualmente a la jerarquía urbana española mucho mejor que otros acercamientos basados en las delimitaciones administrativas. El mapa nacional resulta equilibrado respecto a grandes infraestructuras como el AVE, pudiendo incluso servir de base a otras políticas de distribución territorial (atención sanitaria, educativa, financiación, etc.).

Por último, se adjuntan dos tablas comparativas: una con las 12 principales Grandes Áreas Urbanas (GAUs) de España y sus respectivas áreas metropolitanas según criterios de accesibilidad; y otra contrastando las 6 primeras GAUs con sus respectivas GRMs (ver FIGs. 11 y 12).

\section{Discusión final}

Después de haber repasado los principales retos de nuestras metrópolis, con su triple rompecabezas de caracterización, gobernanza y ordenación territorial, es momento ahora para una somera relación de ideas que contribuyan a un mejor gobierno metropolitano en España. De un lado, pocos dudan de que el deseado encaje entre métodos de cuantificación y mecanismos de gobernanza

\begin{tabular}{|c|c|c|c|c|c|c|c|c|}
\hline \multicolumn{5}{|c|}{ Grandes Áreas Urbanas 2018} & \multicolumn{4}{|c|}{ Áreas Metropolitanas 2019 [10’] } \\
\hline & Mun. & Sup. $\left(\mathrm{km}^{2}\right)$ & № hab. & $\mathrm{Hab} . / \mathrm{km}^{2}$ & Mun. & Sup. $\left(\mathrm{km}^{2}\right)$ & № hab. & $\mathrm{Hab} . / \mathrm{km}^{2}$ \\
\hline Madrid & 52 & $2.890,1$ & 6.055 .116 & 2.095 & $(128)$ & $3.804,8$ & 6.354 .586 & $1.670,15$ \\
\hline Barcelona & 165 & $3.271,5$ & 5.079 .243 & 1.553 & (209) & $2.742,9$ & 5.126 .933 & $1.869,20$ \\
\hline Valencia & 45 & 628,8 & 1.545 .342 & 2.458 & (76) & $1.009,6$ & 1.710 .689 & $1.694,42$ \\
\hline Sevilla & 24 & $1.529,2$ & 1.305 .538 & 854 & (36) & 971,1 & 1.249 .069 & $1.286,19$ \\
\hline Málaga & 8 & 817,4 & 967.250 & 1.183 & (25) & $1.076,1$ & 1.194 .590 & $1.110,16$ \\
\hline Bilbao & 35 & 504,0 & 900.523 & 1.787 & (66) & 806,1 & 957.646 & $1.188,01$ \\
\hline Asturias & 18 & $1.462,9$ & 807.802 & 552 & (26) & $1.167,5$ & 745.446 & 638,52 \\
\hline Zaragoza & 15 & $2.295,4$ & 742.363 & 323 & (33) & 888,4 & 716.670 & 806,66 \\
\hline Alicante-Elche & 6 & 683,3 & 694.902 & 1.017 & (52) & $1.616,9$ & 1.147 .046 & 710,06 \\
\hline Murcia & 10 & $1.230,9$ & 650.468 & 528 & (22) & $1.072,7$ & 690.499 & 643,69 \\
\hline Bahía de Cádiz & 6 & $1.792,0$ & 639.656 & 357 & (10) & 741,5 & 575.240 & 775,82 \\
\hline Vigo-Pontevedra & 14 & 743,2 & 584.507 & 786 & (21) & 616,7 & 437.815 & 709,98 \\
\hline TOTAL & \multicolumn{4}{|c|}{ 19.972.710 } & \multicolumn{4}{|c|}{20.906 .229} \\
\hline
\end{tabular}

FIG. 11/ Las principales Grandes Áreas Urbanas (GAUs) de España y sus áreas metropolitanas propuestas.

Fuente: Elaboración propia a partir de datos de MINISTERIO dE FOMENTO (2018), CIESIN (2017) y FLORCZYK \& al. (2019a).

\begin{tabular}{|c|c|c|c|c|c|c|c|c|}
\hline \multicolumn{5}{|c|}{ Grandes Áreas Urbanas 2018} & \multicolumn{4}{|c|}{ Grandes Regiones Metropolitanas 2019* [30'] } \\
\hline & Mun. & Sup. $\left(\mathrm{km}^{2}\right)$ & № hab. & $\mathrm{Hab} . / \mathrm{km}^{2}$ & Mun. & Sup. $\left(\mathrm{km}^{2}\right)$ & № hab. & $\mathrm{Hab} . / \mathbf{k m}^{2}$ \\
\hline Central (MADRID) & 52 & $2.890,1$ & 6.055 .116 & 2.095 & $(341)$ & $11.294,6$ & 6.966 .348 & 616,78 \\
\hline Catalana (BARCELONA) & 165 & $3.271,5$ & 5.079 .243 & 1.553 & $(787)$ & $17.624,6$ & 7.142 .062 & 405,23 \\
\hline Levante (VALENCIA) & 45 & 628,8 & 1.545 .342 & 2.458 & $(490)$ & $18.064,2$ & 6.040 .993 & 334,41 \\
\hline Suroeste (SEVILLA) & 24 & $1.529,2$ & 1.305 .538 & 854 & $(120)$ & $8.511,6$ & 2.699 .422 & 317,14 \\
\hline Costa del Sol (MÁLAGA) & 8 & 817,4 & 967.250 & 1.183 & (87) & $4.560,1$ & 1.724 .606 & 378,19 \\
\hline Norte (BILBAO) & 35 & 504,0 & 900.523 & 1.787 & $(745)$ & $18.936,4$ & 3.433 .003 & 181,29 \\
\hline TOTAL & \multicolumn{4}{|c|}{15.853 .012} & \multicolumn{4}{|c|}{ 28.006.434 } \\
\hline
\end{tabular}

FIG. 12/ Las 6 primeras GAUs con sus respectivas GRMs. En negrita, los valores representativos más altos. 
pasa por el sometimiento de los primeros a los límites administrativos locales, o bien por la reforma profunda de los segundos. En nuestra opinión, esta última opción no debería dejar de considerar como una alternativa plausible el ajuste de la delimitación local, ya sea por integración municipal o por la aparición de un nuevo modelo territorial surgido de la iniciativa estatal.

En segundo lugar, a nadie se le escapa la necesidad creciente de un aumento de la participación de los ciudadanos en los gobiernos locales, aquellos por los que se siente una mayor proximidad. Las actuales dificultades que conllevan la gobernanza multinivel o incluso la reforma de los mapas locales hacen, no obstante, que el paso de la ciudad a la región metropolitana policéntrica (FARINós DASí \& PeIRó \& Gomis Fons, 2018: 186) deba erigirse como una opción no menos cabal. Frente a los problemas del monocentrismo y del minifundismo insuficiente, convendría apostar por modelos locales en red (¿traspaso de las competencias municipales hacia un órgano supramunicipal legitimado políticamente?), con carácter vinculante y no puramente estratégico, para un ámbito no cerrado ligado a una caracterización objetivable.

$\mathrm{Si}$, además, en ese proceso de delimitación basada en criterios cuantitativos, se vinculara la planificación espacial con la económica -agglomeration wage premium, identificación de local labor markets (FERIA TORIBIO \& CASADO-DIAZ \& MARTÍNEZ BERNABEU, 2015), etc.- la caracterización no sólo sería objetivable, sino que resultaría homogénea y global (MoRENO-MONROY \& VenERI \& SCHIAVINA, 2019). Para ello, y con el fin de facilitar una recopilación estadística más frecuente, quizá deberían plantearse estrategias que facilitasen un acceso de la Administración a patrones de movilidad, por ejemplo, mediante la trazabilidad consentida de vehículos y smartphones.

Como apunte final, cabe decir que la vinculación de los ámbitos de gobernanza metropolitana con repositorios como la UCDB de 2019 (FLORCZYK \& al., 2019a) o el CIESIN, permitiría una mayor transparencia a la hora de revisar la extensión de cada espacio metropolitano. El contexto actual de reflexión sobre el papel de las administraciones puede ser propicio para esta restructuración, si bien la gran cantidad de intereses creados y la dificultad para legislar desde el Estado en cuestiones locales lo complican sobremanera. ¿Se atreverá alguien a ponerle el cascabel a este gato?

Queda, pues, abierto el debate. La batería de propuestas aquí alumbradas pretende, ante todo, aprovechar las ventajas de la colaboración interdisciplinar entre geógrafos, planificadores y economistas, y favorecer su convergencia. Desde el convencimiento de que no hay futuro metropolitano sin territorio, se ha apostado por incidir en los aspectos espaciales (delimitación de áreas y regiones metropolitanas) para sugerir después propuestas de actualización del mapa nacional de las entidades locales.

En todo caso, parece claro que la integración de los ámbitos supramunicipales en un modelo territorial sostenible pasa por nuevas formas de gobernanza, basándose éstas a su vez en criterios de caracterización remota cuantitativa (HERTHOGS \& al, 2019: 100). Esperemos que este esfuerzo por la definición espacial homogénea de los espacios metropolitanos -para los que poco a poco se irá disponiendo de una mayor cantidad de información desagregada, actualizada y en acceso abierto- suponga un paso más hacia la ordenación racional del suelo en nuestro país.

\section{Bibliografía}

Bosker, Maarten \& Jane PARK \& Mark ROBERTs (2018): Definition Matters: Metropolitan Areas and Agglomeration Economies in a Large Developing Country. WPS 8641. Policy Research Working Paper. Washington, D.C.: World Bank Group.

Ciesin (Center For InTERnational EARTH SCIENCE INFOR-MATION NETWORK) (2017): Gridded Population of the World, Version 4 (GPWv4): Population Density Adjusted to Match 2015 Revision UN WPP Country Totals, Revision 10. Palisades, NY: NASA Socioeconomic Data and Applications Center (SEDAC).

[En línea: https://doi.org/10.7927/H49884ZR].

DiJkstRa, Lewis \& Hugo Poelman (2014): A Harmonised Definition of Cities and Rural Areas: The New Degree of Urbanization. Brussels: European Commission.

Duranton, Gilles (2015): "Growing through Cities in Developing Countries", World Bank Research Observer. [En línea: https://doi.org/10.1093/wbro/lku006].

Elinbaum, P. \& Daniel Galland (2016): "Analysing Contemporary Metropolitan Spatial Plans in Europe Through Their Institutional Context, Instrumental Content and Planning Process", European Planning Studies 24 (1) 1-26. [En línea: https://doi.org/10.1080/09654313.2015.1036843].

European Commission (2018): Atlas of the Human Plane 2018 - A World of Cities. Luxembourg: Publications Office of the European Union.

[En línea: https://doi.org/10.2760/124503]

EUROPEAN UNION (2016): Urban Europe. Statistics on Cities, Towns and Suburbs: 2016 Edition. Urban Europe. Statistics on Cities, Towns and Suburbs. Luxemburgo: Publications Office of the European Union. [En línea: https://doi.org/10.2785/91120].

FARINós DAsí, Joaquín \& al. (2017): "Situación y Evolución de La Política de Ordenación Del Territorio en Los Gobiernos y Administraciones de Las CCAA", en XXV Congreso de La Asociación de Geógrafos Españols (AGE). Naturaleza, Territorio y Ciudad En Un Mundo Global. Madrid

FARINós Dasí, Joaquín \& Enrique PeIRÓ \& Andrés Gomis Fons (2018): "Planificación y Ordenación Territorial en Espacios Metropolitanos. Evolución y Análisis de La Situación en España", en Cultura Territorial e Innovación Social: ¿Hacia Un Nuevo Modelo Metropolitano en 
Europa del Sur?, Nacima BARON-Yelles \& Joan Romero GonZÁlez (eds.): 157-92. Valencia: PUV, Publicaciones de la Universidad de Valencia.

FERIA TORIBIO, José María (2018): "Crecimiento Urbano, Crisis Inmobiliaria y Planificación Metropolitana En España." Ciudad y Territorio. Estudios Territoriales L (198): 651-69.

- \& José Manuel Casado Díaz \& Lucas Martínez Bernabeu (2015): "Inside the Metropolis: The Articulation of Spanish Metropolitan Areas into Local Labor Markets", Urban Geography 36 (7): 1018-41. [En línea: https://doi.org/10.1080/02723638.2015.1053199].

- \& Lucas Martínez Bernabeu (2016): "La Definición y Delimitación del Sistema Metropolitano Español: Permanencias y Cambios entre 2001 y 2011", Ciudad y Territorio: Estudios Territoriales, XLVIII (187): 9-24.

- \& Gwendoline DE Oliveira Neves \& Claudia Hurtado RodRíguez (2018): "Une Méthode pour la Délimitation des Aires Métropolitaines. Application au Système Urbain Espagnol", Cybergeo : European Journal of Geography (852). [En línea: https://doi.org/10.4000/cybergeo.29101]

FLoRCZYK, Aneta \& al. (2019a): GHS Urban Centre Database 2015, multitemporal and multidimensional attributes, R2019A. European Commission, Joint Research Centre. [En línea:

http://data.europa.eu/89h/53473144-b88c-44bcb4a3-4583ed1f547e]

- \& al. (2019b): "The Generalised Settlement Area: mapping the Earth surface in the vicinity of built-up areas", International Journal of Digital Earth. [En línea: https://doi.org/ 0.1080/17538947.2018.1550121]

GóMEZ GIMÉNEZ, José Manuel (2017): "El área urbana funcional de Madrid (1991-2011). Metodología y resultados de una propuesta de delimitación y caracterización multicriterio", Territorios en Formación 11: 3-23. [En línea: https://dx.doi.org/10.20868/tf.2017.11.3457].

Herthogs, Pieter \& al. (2019): "Big Data Informed Urban Design and Governance", en Future Cities Laboratory Indicia 02, Stephen CAIRNS \& Devisari TunAs (eds.): 99-110. Zurich: Lars Müller Publishers.

HILDENBRAND, Andreas (2017): "El Abandono de la Cuestión Metropolitana en España”, Revista Iberoamericana de Urbanismo 13: 25-46.

[En línea: http://hdl.handle.net/2117/108615]

HORNER, Mark W. (2004): "Spatial Dimensions of Urban Commuting: A Review of Major Issues and Their Implications for Future Geographic Research", The Professional Geographer 56 (2): 160-73.[En línea: https://doi.org/10.1111/j.0033-0124.2004.05602002.x].

Instituto GeOGRÁfICo NACIONAL (2018): España en Mapas: Una Síntesis Geográfica. Madrid: Centro Nacional de Información Geográfica.

[En línea: https://doi.org/10.7419/162.06.2018]

MELCHIORRI, Michele \& (2018): "Unveiling 25 Years of Planetary Urbanization with Remote Sensing: Perspectives from the Global Human Settlement Layer", Remote Sensing 10 (768).

[En línea: https://doi.org/10.3390/rs10050768]

Metrex (2014): "Metropolitan Dimension. Position Statement." Glasgow: The Network of European Metropolitan Regions and Areas.

MEYERS, Gereon \& Susan SHAHEEN (eds.) (2017): Disrupting Mobility: Impacts of Sharing Economy and Innovative Transportation on Cities. Springer International Publishing. [En línea:

http://www.springer.com/978-3-319-51601-1]

MinISTERIO DE FOMENTO (2018): "Atlas Urbanas en España 2018. Constitución, Cuarenta Años de las Ciudades Españolas". Madrid.
Moreno-Monroy, Ana Isabel \& Paolo VenerI \& Marcello SCHIAVINA (2019): "Urban Agglomerations in the world. Revisiting suburbanization trends through coherent city definitions", en $21^{\text {st }}$ European Colloquium on Theoretical and Quantitative Geography. 5-9 September, Mondorf-les-Baines, Luxemburgo.

PESARESI, Martino \& Sergio FrEIRE (2016): "GHS Settlement Grid Following the REGIO Model 2014 in Application to GHSL Landsat and CIESIN GPW V4-Multitemporal (1975-1990-2000-2015)". [En línea: http://data.europa. eu/89h/irc-ghsl-ghs smod pop globe r2016a].

— \& al. (2016): "Atlas of the Human Planet 2016. Mapping Human Presence on Earth with the Global Human Settlement Layer." Luxemburgo: Publications Office of the European Union.

[En línea: https://doi.org/10.2788/582834].

RoDE, Philipp \& Nuno F. DA CRUZ (2018): "Governing Urban Accessibility: Moving beyond Transport and Mobility", Applied Mobilities 3 (1): 8-33. [En línea: https://doi.org/10.1080/23800127.2018.1438149].

Romaní, Javier \& José Manuel CASADO-Díaz \& Adelaida LILLO-BAÑULS (2017): "Explorando las Relaciones entre el Desplazamiento al Trabajo y los Cambios de Residencia en España. Un Análisis con Datos Individuales", Papers 102 (4): 705-25.

[En línea: https://doi.org/10.5565/rev/papers.2417].

SABO, Fili; \& al. (2018): "Comparison of Built-up Area Maps Produced within the Global Human Settlement Framework", Transactions in GIS 00: 1-31. [En línea: https://doi.org/10.1111/tgis.12480]

SENNETT, Richard \& Ricky BURDETT \& Saskia SASSEN (2018): "The Quito Papers and the New Urban Agenda". New York: Routledge.

SMITH, Duncan A. (2017): "Visualising World Population Density as an Interactive Multi-Scale Map Using the Global Human Settlement Population Layer", Journal of Maps 13 (1): 117-23. [En línea: https://doi.org/10.1080/17445647.2017.1400476].

UCHIDA, Hirotsugu \& Andrew Nelson (2011): "Agglomeration Index: Towards a New Measure of Urban Concentration", en Urbanization and Development: Multidisciplinary Perspectives, Jo BEALL; Basudeb GuHA-KhASNOBIS, and Ravi Kanbur (eds.). Oxford Scholarship Online. [En línea: https://doi.org/10.1093/acprof:oso/9780199590148.0 03.0003].

United Nations (2018): World Urbanization Prospects: The 2018 Revision, Methodology. Working Paper No. ESA/P/WP.252. New York: UN Department of Economic and Social Affairs - Population Division.

WEISS, D. J. \& al. (2018): "A Global Map of Travel Time to Cities to Assess Inequalities in Accessibility in 2015", Nature 553 (January): 333-6.

[En línea: https://doi.org/10.1038/nature25181]

\section{Abreviaturas}

ANE - Atlas Nacional de España

AUE - Agenda Urbana Española

CIESIN - Center for International Earth Science Information Network

CNIG - Centro Nacional de Información Geográfica

ESA - European Space Agency

FUA - Functional Urban Area

GAU - Grandes Áreas Urbanas

GRM - Grandes Regiones Metropolitanas

GHS-BU - Global Human Settlement, Built-Up

GHS-POP - Global Human Settlement, Population

GHS-SMOD - Global Human Settlement, Settlement Mode 


\section{Estudios}

GHS-UCDB - Global Human Settlement, Urban Center Database GHSL - Global Human Settlement Layer GPW - Gridded Population of the World HDC - High-Density Clúster

INE - Instituto Nacional de Estadística

JRC - Joint Research Center

LAU - Local Administrative Unit

METREX - Network of European Metropolitan Regions and Areas

NASA - National Aeronautics and Space Administration
La cuantificación metropolitana en España

Juan Ramón Selva-Royo

NOAA - National Oceanic and Atmospheric Administration OCDE - Organización para la Cooperación y el Desarrollo Económico

SEDAC - Socioeconomic Data and Applications Center

SML - Symbolic Machine Learning

S-MOD - Settlement Model

UC - Urban Center

UCDB - Urban Center Database

UN DESA - United Nations Department of Economic and Social Affairs 